\title{
È possibile predire nell'infanzia le capacità scolastiche e di linguaggio ad 11 anni: i risultati di uno studio di coorte in Australia
}

Eadie P, Bavin EL, Bretherton L, et al.

Predictors in Infancy for Language and Academic Outcomes at 11 Years

Pediatrics. 2021;147(2):e20201712

Le competenze precoci di linguaggio sono fondamentali per l'apprendimento prescolare e $i$ successivi risultati scolastici, tuttavia non è chiaro in che momento sia possibile predire precocemente in modo ottimale le difficoltà successive, per un intervento tempestivo. Questo studio australiano ha seguito 1.910 bambini dalletà di 7.5-10 mesi, raccogliendo dati su 12 fattori di rischio associati allo sviluppo del linguaggio. È stato analizzato un sottogruppo di 839 bambini con follow-up completo a 11 anni, valutazione del linguaggio a 2, 4, 5, 7 anni e valutazione dei risultati scolastici con test nazionali a 8, 10 e 12 anni. I fattori precoci di rischio sono risultati buoni predittori dei risultati scolastici a 11 anni. La competenza di linguaggio a 4 anni è risultata essere il miglior predittore degli esiti di linguaggio a 11 anni. Si conferma la necessità di un supporto precoce allambiente familiare in presenza di fattori di rischio e si individua nell'età di 4 anni il momento migliore per decidere la necessità di un intervento sullo sviluppo del linguaggio.

It is possible to predict school and language skills at age of 11: the results of a cohort study in Australia

Early language skills are essential for preschool learning and subsequent school results, however it is not clear at what time it is possible to predict the subsequent difficulties in an optimal way ear$l y$, for a timely intervention. This Australian study followed 1.910 children from the age of 7.5-10 months, collecting data on 12 risk factors associated with language development. A subgroup of 839 children was analyzed with full follow-up at 11 years, language assessment at 2, 4, 5, 7 years and evaluation of school results with national tests at 8,10 and 12 years. Early risk factors were good predictors of school achievement at 11. Language proficiency at 4 years was the best predictor of language outcomes at 11 years. The need for early support to the family environment in the presence of risk factors is confirmed and the best time to decide the need for intervention on language development is identified at the age of 4 .

\section{Metodo}

\section{Obiettivo (con tipo studio)}

Esaminare il ruolo dei fattori di rischio della prima infanzia e delle abilità linguistiche acquisite in età prescolare e scolare (2, 4, 5 e 7 anni) nel determinare i risultati scolastici e linguistici di bambini di 11 anni. Studio retrospettivo di coorte.

\section{Popolazione}

839 di 1.910 bambini reclutati tra i 7.5 ed i 10 mesi di vita, da uno studio prospettico di coorte (The Early Language in Victoria Studi - ELVS) provenienti da 6 diverse aree governative della città metropolitana di Melbourne, nello stato di Victoria, Australia, rappresentativi dell'intero spettro socioeconomico definito secondo l'Australian Indexes of Disadvantages (SEIFA).

I bambini della coorte ELVS, inclusi in questo studio, avevano completato 11 anni di raccolta dati, le valutazioni linguistiche a 2, 4, 5 e 7 anni ed i test del "Programma Valutazione Nazionale - alfabetizzazione e calcolo" (NAPLAN) in uno o tutti i livelli scolastici: classe 3, 5 e 7, all'età di circa 8, 10 e 12 anni.

\section{Esposizione}

12 fattori di rischio associati allo sviluppo del linguaggio che includevano variabili sociodemografiche inerenti il bambino (sesso, gemellarità, ordine di genitura, prematurità, peso alla nascita), la madre (età, salute mentale, istruzione, lessico) e la famiglia (lingua diversa dall'inglese comunemente parlato-NESB-, status socioeconomico -SES-, storia familiare di difficoltà di linguaggio e/o espressione). I dati sui 12 fattori di rischio associati allo sviluppo del linguaggio sono stati raccolti da studi precedenti su questa coorte, rilevati a 8-10 mesi, 2, 4 e 7 anni.

\section{Outcome/Esiti}

Competenze linguistiche ed accademiche a 11 anni. Le competenze linguistiche sono state misurate utilizzando il CELF-4 e definite basse quando i punteggi in termini di ricezione o espressione erano inferiori di 1.25 DS rispetto alla media. I risultati accademici sono stati rappresentati dal NAPLAN di grado 7 per lettura, grammatica e punteggiatura. Scarse capacità accademiche erano definite da punteggi inferiori di 1.25 DS rispetto alla media dello stato di Victoria. I risultati accademici sono stati riportati separatamente per i bambini con linguaggio normale e per quelli con punteggi linguistici bassi a 11 anni. I punteggi NAPLAN di ciascun gruppo erano stimati con un intervallo di confidenza (IC) del 95\% ai gradi 3, 5 e 7, e le percentuali sono state confrontate utilizzando una regressione logistica non variabile.

\section{Tempo}

Lo studio di coorte ELVS è cominciato nel 2002; il reclutamento si è svolto tra Settembre 2003 e Aprile 2004. I bambini sono stati valutati a partire dal 2002, all' età di 7.5-10 mesi, sino all'età di 11 anni. 


\section{Risultati principali}

I fattori a cui è esposto un bambino nella prima infanzia, considerati insieme, spiegano l' $11 \%$ ed il $12 \%$ di varianza nei punteggi inerenti rispettivamente il linguaggio ricettivo ed espressivo a 11 anni. L'analisi di regressione lineare seleziona 4 dei 12 fattori: ordine di genitura, istruzione materna, lessico materno e storia familiare di difficoltà di linguaggio. L'analisi fatta mediante regressione logistica seleziona ulteriormente ed individua nell'istruzione materna l'unico fattore di rischio comune in grado di predire l'abilità linguistica e discriminare tra capacità linguistiche normali o basse. A 11 anni, le probabilità di avere un linguaggio ricettivo basso risultano infatti inferiori in presenza di una maggiore istruzione materna (OR 0.45; IC 95\% 0.22, 0.94] e le probabilità di avere un basso livello linguistico espressivo sono minori in presenza di un grado di istruzione materna superiore (OR 0.37; IC 95\% 0.17, 0.79). Le probabilità di avere un basso livello linguistico espressivo sono minori anche per le ragazze (OR 0.46; IC 95\% 0.25, 0.84) e per i gemelli (OR 5.05; IC $95 \% 1.59,15.97)$. La capacità predittiva aumenta quando nell'analisi ai fattori familiari vengono aggiunti i punteggi linguistici acquisiti dai 2 ai 7 anni, e di maggior impatto risulta il punteggio linguistico registrato a 4 anni. Per quanto riguarda le abilità accademiche a 11 anni, gli stessi fattori della prima infanzia spiegano una varianza del $13 \%$ e $14 \%$ dei punteggi rispettivamente nella lettura e nella grammatica e punteggiatura al grado 7 (II ${ }^{\circ}$ media inferiore), con aumenti al $43 \%$ e $54 \%$ quando vengono inclusi i punteggi linguistici acquisiti da 2 a 11 anni. I fattori della prima infanzia discriminano adeguatamente tra bambini con punteggi linguistici normali e bambini con punteggi linguistici bassi, ma sono ancora più incisivi nel discriminare tra bambini con risultati scolastici normali e bambini con scarso rendimento scolastico. Quando ai modelli dei fattori della prima infanzia sono aggiunti i punteggi linguistici a 4, 5, 7 e 11 anni il valore di area sotto la curva (AUC) aumenta a 0.9 ed oltre.

\section{Conclusioni}

I risultati linguistici dei bambini all'età di 11 anni sono predetti con precisione dalle loro capacità linguistiche a 4 anni.

I risultati accademici sono invece predetti dai fattori di rischio precoci familiari e dell'ambiente domestico. La scarsa capacità linguistica a 11 anni è associata a scarse abilità accademiche. I bambini con scarse abilità linguistiche all'età di 11 anni hanno ottenuto punteggi significativamente inferiori nelle valutazioni nazionali di alfabetizzazione e matematica.

\section{Altri studi sull'argomento}

Uno studio condotto in North Carolina e pubblicato nel 1998 ha esaminato l'utilità di un modello di rischio cumulativo per prevedere i risultati cognitivi e linguistici in un campione di 83 bambini afroamericani di 12 mesi, biologicamente normali che frequentavano i centri di assistenza all'infanzia locali [1]. Sono stati utilizzati dieci fattori di rischio sociali e familiari per costruire un indice di rischio cumulativo: stato di povertà, istruzione materna inferiore alla scuola superiore, dimensioni della famiglia, madre non sposata, eventi di vita stressanti, depressione materna, interazioni madre-bambino, QI, qualità dell'ambiente domestico e qualità dell'ambiente diurno. Le misurazioni dei risultati includevano l'indice di sviluppo mentale della Bayley Scales of Infant Development (MDI, scala progettata per valutare la cognizione attraverso la valutazione della percezione sensoriale, della conoscenza, della memoria, della risoluzione dei problemi e del linguaggio precoce, misura una combinazione di sviluppo cognitivo e del linguaggio precoce), il punteggio totale dalla Communication and Symbolic Behavior Scale (CSBS, test standardizzato progettato per valutare neonati e bambini in età prescolare che sono a rischio di ritardi nella comunicazione) e il punteggio Receptive Communication Age dal Sequenced Inventory for Communication Development-Revised (SICD-RCA valuta discriminazione, consapevolezze e comprensione del suono e del linguaggio e inoltre misura le capacità espressive). I risultati hanno indicato che l'indice di rischio cumulativo era significativamente correlato con CSBS e SICD-RCA, ma non con l'MDI, tenendo conto di modeste quantità di varianza. L'impatto del rischio ambientale sullo sviluppo cognitivo e linguistico dei bambini è stato valutato in uno studio inglese longitudinale di 78 famiglie ad alto rischio, pubblicato nel 2008 [2]. I fattori di rischio esaminati erano lo stato sociale della famiglia, la condizione psicosociale della madre e la qualità del coinvolgimento della coppia madre-bambino a 1 anno di vita (interazione e attaccamento madre-bambino). Le misure degli esiti includevano la Bayley MDI (a 24 mesi) e la Preschool Language Scale (a 36 mesi). I dati hanno rilevato che il coinvolgimento diadico è stato un mediatore importante nella relazione tra rischio ambientale e successivo sviluppo delle competenze del bambino. All'interno di un quadro di fattori di rischio e fattori di protezione sono state valutate relazioni specifiche tra le prime esperienze interattive, la sicurezza dell'attaccamento infantile e il successivo guadagno cognitivo e linguistico. I risultati hanno rilevato che un attaccamento sicuro può essere considerato un fattore protettivo. Nello studio di Sohr-Preston et al. è stata considerata sia la tempistica della depressione materna che la cronicità della depressione delle madri sul rischio di poter avere nei bambini ritardi cognitivi e linguistici [3]. Tale indagine ha evidenziato che l'esposizione dei bambini alla depressione materna cronica sembra essere associata a esiti più problematici per i bambini, forse perché la depressione interferisce con la capacità delle madri di rispondere in modo sensibile e coerente nel tempo. In accordo con questa aspettativa, è stato riscontrato che gli interventi mirati alle pratiche genitoriali delle madri depresse aumentano la competenza cognitiva dei bambini durante la prima infanzia.

\section{Che cosa aggiunge questo studio}

La valutazione delle abilità linguistiche e un eventuale intervento di sostegno linguistico potrebbero essere avviati già l'anno prima dell'inizio della scuola e che i bambini con un basso rendimento scolastico possono essere adeguatamente e precocemente identificati attraverso alcuni fattori di rischio rilevati in età prescolare. Questi risultati rappresentano inoltre un potenziale per ricerche future che si focalizzino sull'impatto che una preparazione pre-scolare e scolare di alta qualità può avere nel migliorare la vulnerabilità educativa di bambini con scarse abilità linguistiche. 


\section{Commento}

\section{Validità interna}

Disegno dello studio: popolazione ed esposizione sono ben definite. I fattori di rischio sono chiaramente definiti, gli strumenti di valutazione delle abilità linguistiche ed accademiche nelle varie età sono universalmente riconosciuti ed adattati (con autorizzazione) per l'Australia. Lo studio presenta dei limiti: la coorte reclutata non è infatti rappresentativa della popolazione di interesse, in quanto a 11 anni i partecipanti erano in maggioranza femmine, vivevano in aree meno svantaggiate, avevano madri di età maggiore con livello di vocabolario e scolastico superiore rispetto ai non partecipanti. Inoltre vi erano meno famiglie NESB; la capacità dei fattori di rischio precoci nel predire da soli ed efficacemente le scarse abilità scolastiche ad 11 anni potrebbe dipendere dal fatto che non è stata inclusa nello studio la valutazione di una variabile intermedia delle capacità accademiche precoci, a differenza di quanto è stato fatto per il linguaggio; il NAPLAN è infatti somministrato a partire dall'età scolare. Altri fattori che possono influenzare lo sviluppo del linguaggio come le esperienze sfavorevoli infantili, la salute mentale dei genitori, la frequenza ad asili o scuole dell'infanzia non sono stati valutati. Esiti: rilevanti e la durata del follow up è sufficiente a permettere la comparsa degli esiti di interesse.

Conflitto di interesse: nessuno.

\section{Trasferibilità}

Popolazione studiata: quella inclusa non è la popolazione completamente rappresentativa della realtà australiana sia per condizione sociale che per composizione etnica; pertanto si deve tener conto di questo elemento per valutare la trasferibilità nella popolazione italiana. .

Tipo di intervento: importante anche per la nostra realtà, ma i test effettuati dovrebbero essere definiti in base alla nostra lingua e ai programmi didattici nazionali. La valutazione dei fattori di rischio dovrebbe essere il primo passo necessario di un intervento atto a sostenere lo sviluppo del linguaggio nella popolazione pediatrica vulnerabile già prima dell'inizio della scuola dell'obbligo.

1. Hooper SR, Burchinal MR, Roberts JE, et al. Social and family risk factors for infant development at one year: An application of the cumulative risk model. Journal of Applied Developmental Psychology. 1998;19(1):85-96.

2. Morisset CE, Barnard KE, Greenberg MT, et al. Environmental influences on early language development: The context of social risk. Development and Psychopathology. 1990;2(2):127-49.

3. Sohr-Preston SL, Scaramella LV. Implications of timing of maternal depressive symptoms for early cognitive and language development. Clin Child Fam Psychol Rev. 2006;9(1):65-83.

Scheda redatta dal gruppo di lettura di Milano:

Riccardo Cazzaniga, Valeria D’Apolito, Valentina Decimi, Gian Piero Del Bono, Lucia Di Maio, Elena Groppali, Laura Gualtieri, Laura Martelli, Maria Luisa Melzi, Maddalena Migliavacca, Aurelio Nova, Francesco Peia, Maria Antonietta Pelagatti, Ambrogina Pirola, Ferdinando Ragazzon, Giulia Ramponi, Patrizia Rogari, Claudio Ronconi, Alessandra Sala, Martina Saruggia, Federica Zanetto. 